\title{
CDX2 expression is concordant between primary colorectal cancer lesions and corresponding liver metastases independent of chemotherapy: a single-center retrospective study in Japan
}

\author{
Yasuyuki Shigematsu ${ }^{1}$, Kentaro Inamura ${ }^{1}$, Yoshihiro Mise $^{2}$, Akio Saiura ${ }^{2}$, Emil \\ Rehnberg $^{3}$, Noriko Yamamoto ${ }^{1}$, Yuichi Ishikawa ${ }^{1}$, Shunji Takahashi ${ }^{4}$ and Hiroaki \\ Kanda $^{1}$ \\ ${ }^{1}$ Department of Pathology, The Cancer Institute of Japanese Foundation for Cancer Research (JFCR), Koto, Tokyo 135-8550, \\ Japan \\ ${ }^{2}$ Division of Gastroenterology Center, The Cancer Institute Hospital, JFCR, Koto, Tokyo 135-8550, Japan \\ ${ }^{3}$ Division of Business Intelligence, Labs BI, Shanghai 200040, China \\ ${ }^{4}$ Division of General Oncology, The Cancer Institute Hospital, JFCR, Koto, Tokyo 135-8550, Japan \\ Correspondence to: Yasuyuki Shigematsu, email: yasuyuki.shigematsu@jfcr.or.jp \\ Keywords: CDX2; heterogeneity; colorectal cancer; chemotherapy; liver metastasis \\ Received: June 05, $2017 \quad$ Accepted: February 28, $2018 \quad$ Published: March 30, 2018 \\ Copyright: Shigematsu et al. This is an open-access article distributed under the terms of the Creative Commons Attribution Li- \\ cense 3.0 (CC BY 3.0), which permits unrestricted use, distribution, and reproduction in any medium, provided the original author \\ and source are credited.
}

\section{ABSTRACT}

Objective: Loss of caudal-type homeobox transcription factor 2 (CDX2) expression in colorectal cancers (CRCs) has recently been proposed as a promising predictive biomarker for not only prognosis but also response to chemotherapy. However, the relationship between alterations in CDX2 expression during cancer progression and response to chemotherapy remains unclear. We herein aimed to determine the concordance of CDX2 expression between primary CRCs and corresponding liver metastases, in association with chemotherapy.

Results: Primary CRCs exhibited heterogeneous CDX2 expression. Seven of the 144 CRCs in the cohort (4.9\%, 95\% confidential interval, $2.0 \%-9.8 \%)$ were CDX2negative. The concordance rate of the CDX2 expression status in patients who did not receive chemotherapy was $100 \%(P=0.041)$, whereas the concordance rate among patients who received chemotherapy only after primary resection was $96.3 \%(P=$ $0.005)$. Moreover, the concordance rate in patients who received chemotherapy before both primary resection and liver metastasectomy was $100 \%(P<0.001)$.

Conclusion: CDX2 expression status was highly concordant between primary CRCs and corresponding liver metastases, independent of chemotherapy, suggesting that the CDX2 expression status in CRCs was not affected by metastasis or chemotherapy.

Methods: A total of 144 consecutive patients with CRC who were treated at a single center in Japan between 2006 and 2014 were included. Formalin-fixed paraffinembedded whole sections of surgically resected primary CRCs and corresponding liver metastases were assessed for CDX2 expression by immunohistochemistry.

\section{INTRODUCTION}

Caudal-type homeobox transcription factor 2 (CDX2) regulates gut epithelial development and maturation [1-4] and is expressed within nuclei of intestinal epithelial cells from proximal duodenum to distal rectum [5]. Increased CDX2 expression, which is observed in approximately $90 \%-95 \%$ of colorectal adenocarcinomas $[6,7]$, is considered to be a highly sensitive and specific diagnostic marker for adenocarcinomas of intestinal origin [8-10]. In addition to its diagnostic utility, CDX2 is reported to exert tumor-suppressor functions by controlling a number of genes involved in proliferation, migration, and carcinogenesis [11]. However, alterations in the CDX2 
expression during cancer progression have not been examined extensively. The possibility remains that genetic diversification and clonal selection might result in changes in CDX2 expression status [12-15].

Several retrospective studies suggested that the loss of CDX2 expression in colorectal cancers (CRCs) was associated with worse prognosis as well as several adverse prognostic variables, such as high histologic grade, BRAF mutations, and $\mathrm{CpG}$ island methylator phenotype positivity [16-18]. Interestingly, in early-stage CRCs, adjuvant chemotherapy could decrease recurrence risk in patients with loss of CDX2 expression, suggesting that this phenotype might be a potential predictive marker for response to chemotherapy [16]. However, in metastatic $\mathrm{CRCs}$, the loss of CDX2 expression was reported to be associated with lower chemotherapy efficacy [19]. Therefore, it remains unclear whether the CDX2 expression status represents a chemosensitive phenotype.

Chemotherapy plays an important role in the treatment of CRCs. Patients with technically unresectable metastatic CRCs benefit from chemotherapy, which was shown to shift the clinical stage toward a technically resectable condition [20]. Patients with stage III and high-risk stage II CRCs also benefit from adjuvant chemotherapy, as shown by decreased risk of recurrence [21]. In this context, the impact of chemotherapy on CDX2 expression status, which is considered as a promising prognostic marker in CRCs, remains poorly understood, although the expressions of several genes were previously shown to be affected by chemotherapy $[12,22]$.

There is a concern about the presence or absence of heterogeneity of CDX2 expression in CRCs because there are few reports regarding CDX2 expression heterogeneity, and the previous studies [16-18] were conducted using tissue microarrays (TMAs), whose results can be affected by the heterogeneity of the target protein expression. To establish the presence or absence of the heterogeneity of CDX2 expression in CRCs, immunohistochemistry (IHC) using whole sections is necessary.

In the present study, using whole sections of lesions, we aimed to evaluate alterations in CDX2 expression during cancer progression and to assess the impact of chemotherapy on CDX2 expression in primary CRCs and corresponding liver metastases.

\section{RESULTS}

\section{Patient and tumor characteristics}

Demographic and clinicopathological characteristics of all CRC patients are summarized in Table 1. Briefly, at diagnosis, mean patient age was 60.1 years, and most of the patients were male $(68.5 \%)$. A majority of the patients $(80.5 \%)$ had liver metastases at diagnosis, and there was a median of four metastatic liver tumors (These data were not included in Table 1, and partly included in Table 2).
Additionally, a majority of the primary CRCs $(96.5 \%)$ were well or moderately differentiated by histological assessment. A total of 115 patients (79.9\%) had left-sided CRCs. In the present study, 120 out of 144 patients $(83.3 \%)$ received chemotherapy; among these, 82 patients $(68.3 \%)$ received chemotherapy only before metastasectomy, whereas the remaining patients $(31.7 \%)$ received chemotherapy before both primary resection and liver metastasectomy.

\section{Distribution of CDX2 expression in a whole section of primary CRCs and a cut-off value to determine the CDX2 expression status}

To evaluate the expression of CDX2 in CRCs, we performed IHC for CDX2 using whole sections of surgical samples (Figure 1). More than $20 \%$ of primary CRCs showed heterogeneous CDX2 expression (Figure 2). To ensure that the clinical judgment used to determine the CDX2 status of specimens was reproducible, the presence of $50 \%$ CDX2-positive cells within a whole tumor specimen was used as the cut-off point. Specifically, samples were considered as CDX2-positive if 50\% or more of the cancer cells displayed widespread nuclear CDX2 expression, whereas samples were considered as CDX2negative if less than $50 \%$ of the cancer cells showed nuclear CDX2 expression. To assess the robustness of the criterion, concordance between the $\mathrm{CDX} 2$ scoring results obtained by two independent investigators were assessed, which revealed a concordance rate of $97.9 \%$ (95\% confidence interval [CI], 95.5\%-99.2\%; kappa index, 0.82), indicating that the judgment using the criterion was robust.

\section{Clinicopathological characteristics of CDX2- negative CRCs}

Primary CRCs were categorized into CDX2positive and CDX2-negative groups using the defined criteria (Table 2). Therefore, there were seven CDX2negative CRCs $(4.9 \%, 95 \%$ CI, 2.0\%-9.8\%) and 137 CDX2-positive CRCs in the study cohort $(95.1 \%, 95 \% \mathrm{CI}$, $90.2 \%-98.0 \%$ ). Two of the CDX2-negative primary CRCs $(28.5 \%)$ were poorly differentiated adenocarcinomas, compared with three poorly differentiated primary CRCs $(2.2 \%)$ in the CDX2-positive group ( $P=0.02)$, confirming the previous studies reporting that $\mathrm{CDX} 2$-negative CRCs were more likely to be poorly differentiated [18, 23]. In contrast, there were no significant differences in age, sex, the number of liver metastases, or clinical-stage between the CDX2-positive and CDX2-negative groups.

\section{Concordance analysis of the CDX2 expression status between primary CRCs and corresponding liver metastases}

To analyze whether the CDX2 expression status might be affected during the metastatic process, the 
Table 1: Patients and tumor characteristics

\begin{tabular}{|c|c|}
\hline Characteristics & Case No. \\
\hline All cases & 144 \\
\hline Mean age at metastasectomy (years) & 60.1 \\
\hline \multicolumn{2}{|l|}{ Sex } \\
\hline Male & 98 \\
\hline Female & 46 \\
\hline \multicolumn{2}{|l|}{ TNM stage } \\
\hline I & 1 \\
\hline II & 4 \\
\hline III & 23 \\
\hline IV & 116 \\
\hline \multicolumn{2}{|l|}{ Tumor grade } \\
\hline well & 48 \\
\hline $\bmod$ & 91 \\
\hline por & 5 \\
\hline \multicolumn{2}{|l|}{ Tumor location } \\
\hline Cecum & 6 \\
\hline Ascending & 12 \\
\hline Transverse & 11 \\
\hline Descending & 6 \\
\hline Sigmoid & 51 \\
\hline Rectum & 58 \\
\hline \multicolumn{2}{|l|}{ Chemotherapy } \\
\hline Yes & 120 \\
\hline Before metastasectomy only & 82 \\
\hline Before both primary resection and metastasectomy & 38 \\
\hline No & 24 \\
\hline
\end{tabular}

CDX2 expression status of primary CRCs were compared with that of the corresponding liver metastases before chemotherapy (Table 3, patients without chemotherapy). There were 23 CDX2-positive and 1 CDX2-negative primary CRCs. The concordance rate of the CDX2 expression status was $100 \%$, and the association between the CDX2 expression status of metastatic lesions and that of the primary CRC lesions was statistically significant $(P=0.041)$, indicating that the metastatic process did not affect the CDX2 expression status in CRCs.

Using samples from patients who received chemotherapy only after primary resection, we compared the CDX2 expression status between primary CRCs and corresponding metastatic lesions (Table 3, patients with chemotherapy only before metastasectomy). Among a total of $79 \mathrm{CDX} 2$-positive $\mathrm{CRCs}$, the change to $\mathrm{CDX} 2$ negativity was found in only two cases. Conversely, the metastatic lesion in one of the three patients with CDX2negative primary $\mathrm{CRCs}$ that received chemotherapy was CDX2-positive. The concordance rate of the CDX2 expression status was $96.3 \%$, and there was a significant association between the CDX2 expression status of the primary CRCs and metastatic lesions $(P=0.005)$. Therefore, the impact of chemotherapy on CDX2 expression status was considered to be minimal.

The CDX2 expression status of primary CRCs and corresponding metastatic lesions in patients who received chemotherapy before both primary resection and metastasectomy was analyzed (Table 3, patients with chemotherapy before both primary resection and metastasectomy). There were 35 CDX2-positive and 3 CDX2-negative primary CRCs. The concordance rate of the CDX2 expression status was $100 \%$, and there was a significant association in the CDX2 expression status between primary and metastatic lesions among patients receiving chemotherapy $(P=0.0002)$. These data indicated that the CDX2 expression status was not affected by the metastatic process and chemotherapy. 
To confirm that the high concordance can be achieved regardless of the cutoff point, we used a cutoff point of $20 \%$; the numbers of CDX2-positive CRC and CDX2-negative CRC were 115 and 29, respectively, and the overall concordance rate was $95.1 \%$, indicating high concordance (Supplementary Table 1). Therefore, we can conclude that the CDX2 expression status of CRCs is consistent between primary and liver metastatic sites regardless of the cutoff point.

\section{Recurrence-free survival and overall survival among CDX2-positive CRCs and CDX2-negative CRCs}

In an exploratory analysis, we evaluated the association between CDX2 expression and recurrencefree survival (RFS) or overall survival (OS). The analysis revealed that median RFS in the CDX2-positive group was similar to that in the CDX2-negative group (326 days
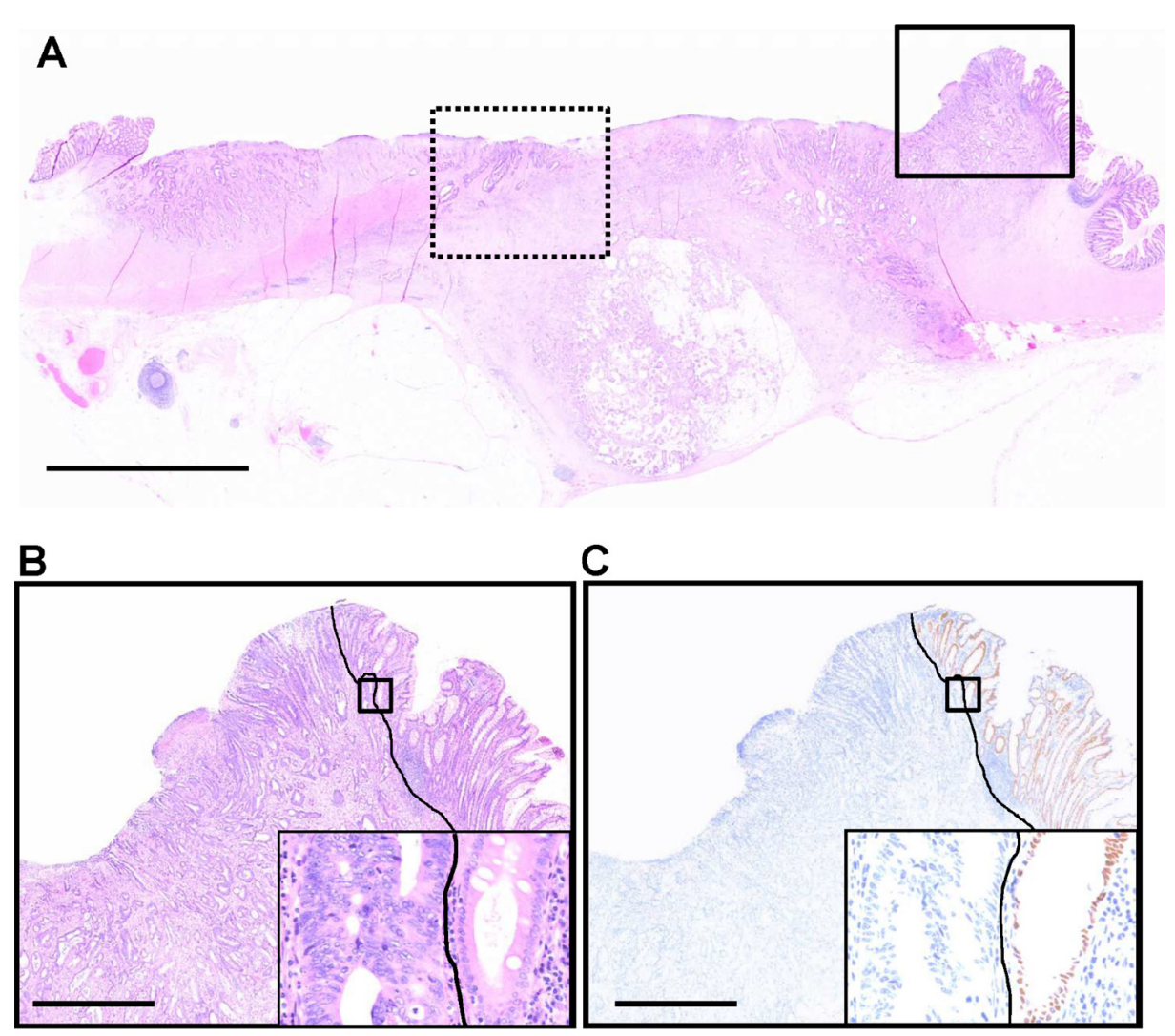

C
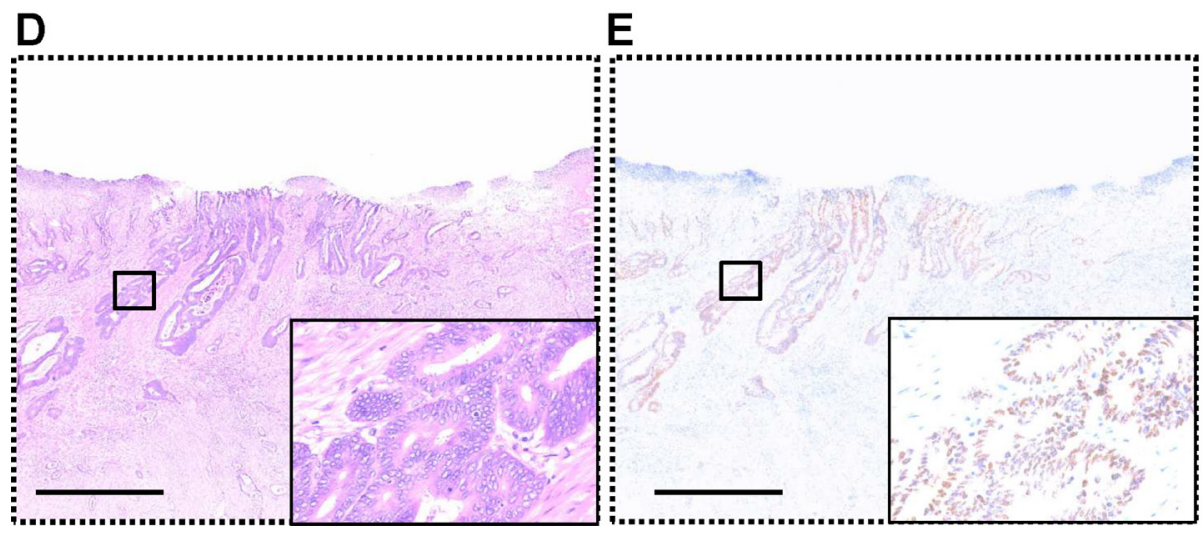

Figure 1: CDX2 expression in a whole formalin-fixed, paraffin-embedded section of a colorectal cancer case, which exhibits heterogeneous CDX2 expression. (A) A representative section stained with hematoxylin and eosin. (B, C) Magnification of the area boxed by the solid line in A. A Solid line represents the boundary between cancerous (left side) and non-cancerous (right side) regions. Insets show higher magnification of areas boxed by thick solid lines. (B) Hematoxylin and eosin staining. (C) Immunohistochemistry (IHC) for CDX2. In the non-cancerous region, colonic epithelial cells with nuclear CDX2 expression serve as positive controls for CDX2 IHC. In contrast, tumor cells in the same region do not express the CDX2. (D, E) Magnification of the area boxed by the dashed line in A. Insets show higher magnification of areas boxed by thick solid lines. (D) Hematoxylin and eosin staining. (E) IHC for CDX2. In the cancerous region, cancer cells exhibit nuclear CDX2 expression. Scale bars, $5 \mathrm{~mm}$ (for A) and $1 \mathrm{~mm}$ (for B, C, D, and E). 
vs. 319 days, $p=0.99$ ) (Supplementary Figure 1A). The three-year OS in the CDX2-positive group was 78\% and that in the CDX2-negative group was 57\%; however, the difference was not statistically significant $(p=0.65)$ (Supplementary Figure 1B).

\section{Concordance analysis of CK7, CK20, CEA, and MUC2 expression status between primary CRCs and corresponding liver metastases}

As a secondary analysis, we investigated the concordance rate of cytokeratin 7 (CK7), CK20, carcinoembryonic antigen (CEA), and mucin 2 (MUC2) expression status. In the same manner as the CDX2 investigation, we used a whole-section of all the samples and set a cutoff point of $50 \%$ of tumor cells to assess whether a tumor is positive or negative for the protein expression. In the primary $\mathrm{CRCs}$, the positivity rates of CK7, CK20, CEA, and MUC2 were 5\%,89\%,85\%, and $3 \%$, respectively. The overall concordance rates of the expression of CK7, CK20, CEA, and MUC2 proteins were $97 \%, 88 \%, 84 \%$, and $98 \%$, respectively (Supplementary Table 2). In patients without chemotherapy $(n=23)$, the concordance rates of the expression of CK7, CK20, CEA, and MUC2 proteins were 96\%, 87\%, 91\%, and 100\%, respectively. In patients who received chemotherapy before metastasectomy only $(n=76)$, the concordance rates of the expression of CK7, CK20, CEA, and MUC2 proteins were $97 \%, 92 \%, 86 \%$, and $97 \%$, respectively. In patients who received chemotherapy before both primary and metastatic resections $(n=35)$, the concordance rates of the expression of $\mathrm{CK} 7, \mathrm{CK} 20, \mathrm{CEA}$, and MUC2 proteins were $97 \%, 83 \%, 77 \%$, and $100 \%$, respectively. Liver metastatic sites also showed high concordance rates with the primary CRCs for CK7, CK20, CEA, and MUC2 expression status.

\section{Expression status of CDX1 in the primary CRCs and its concordance between primary and liver metastatic sites}

As a secondary analysis, we investigated CDX1 expression status in non-cancerous and cancerous tissues using 134 primary CRCs because CDX1 has high structural homology to CDX2 and plays complementary roles in the adult intestine [24], and its expression has been reported to inhibit colon cancer cell proliferation in vitro $[25,26]$. In the primary sites, the nuclear expression of CDX1 was observed in the non-cancerous epithelium. In contrast to the non-cancerous epithelium, the CDX1 expression of cancerous tissue was usually very low or absent (Supplementary Figure 2). In the same manner as the CDX2 investigation, we set a cutoff point of $50 \%$ tumor cells to assess whether a tumor is positive or negative for CDX1 protein expression. Among the 134 primary CRCs, 7 (5.2\%) were CDX1-positive CRCs and 127 (94.8\%) were CDX1-negative CRCs.

As for the concordance analysis, the overall concordance rate of CDX1 expression was $96.3 \%$. In patients without chemotherapy $(n=23)$, the concordance rate was $100 \%$. In patients who received chemotherapy before metastasectomy only $(n=35)$, the concordance rate was $85.7 \%$. In patients who received chemotherapy before both primary and metastatic resections $(n=76)$,

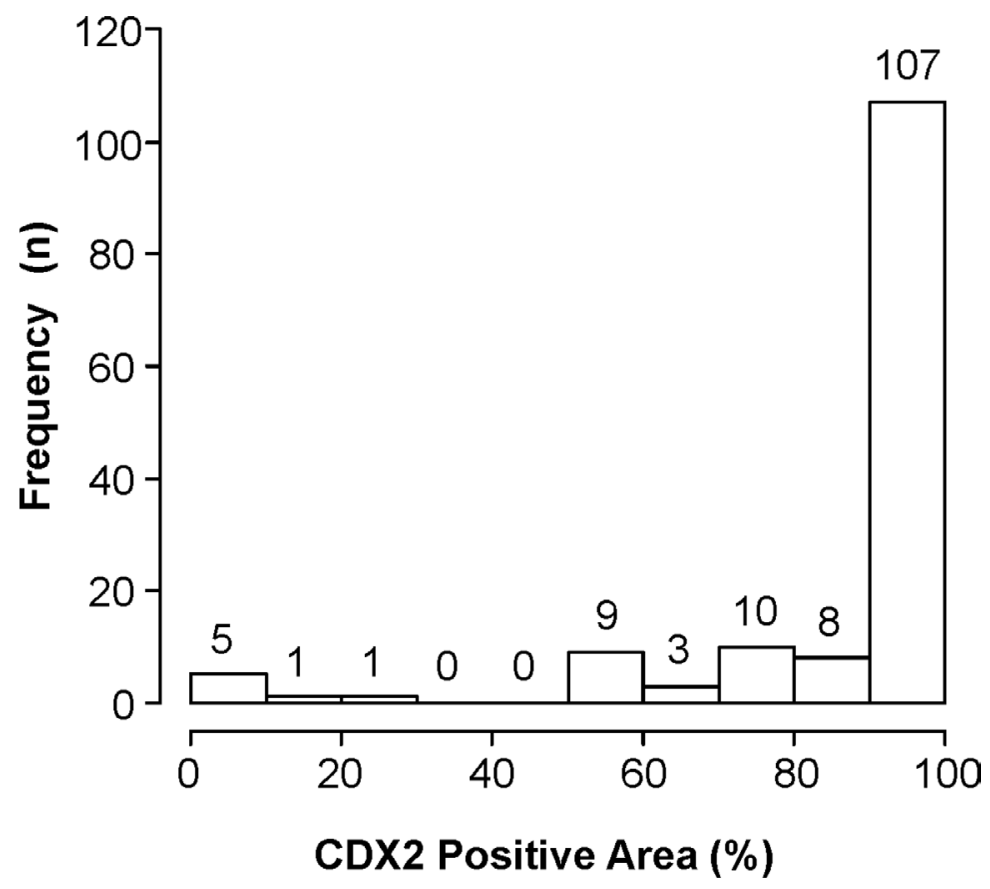

Figure 2: Histogram depicting the ratios of CDX2-positive cells to all cancer cells in whole sections of primary colorectal cancers. In the study cohort, more than 32 cases (22.2\%) showed heterogeneous CDX2 expression. 
Table 2: Relationship between clinicopathological characteristics and CDX2 expression in primary colorectal cancers

\begin{tabular}{|c|c|c|c|}
\hline \multirow[t]{2}{*}{ Characteristics } & \multicolumn{2}{|c|}{ CDX2 expression } & \multirow[t]{2}{*}{$P$-value } \\
\hline & Positive & Negative & \\
\hline All cases & 137 & 7 & \\
\hline \multicolumn{4}{|l|}{ Age } \\
\hline$\leq 65$ & 97 & 5 & 1.00 \\
\hline $65<$ & 40 & 2 & \\
\hline \multicolumn{4}{|l|}{ Sex } \\
\hline Female & 45 & 1 & 0.43 \\
\hline Male & 92 & 6 & \\
\hline \multicolumn{4}{|l|}{ Histology } \\
\hline well & 47 & 1 & 0.02 \\
\hline $\bmod$ & 87 & 4 & \\
\hline por & 3 & 2 & \\
\hline \multicolumn{4}{|c|}{ Number of liver metastases } \\
\hline$<5$ & 84 & 3 & 0.44 \\
\hline $5 \leq$ & 53 & 4 & \\
\hline \multicolumn{4}{|l|}{ Clinical stage } \\
\hline I & 1 & 0 & 0.23 \\
\hline II & 4 & 0 & \\
\hline III & 20 & 3 & \\
\hline IV & 112 & 4 & \\
\hline
\end{tabular}

the concordance rate was $100 \%$. Because of the high concordance rates, we can conclude that CDX1 expression status of CRCs is consistent between primary and liver metastatic sites regardless of metastatic process and chemotherapy.

\section{DISCUSSION}

The present study demonstrated the heterogeneity of CDX2 expression in whole CRC sections. Additionally, the results revealed the high concordance rate of $\mathrm{CDX} 2$ expression between primary CRCs and corresponding liver metastases, which was not affected by chemotherapy. To the best of our knowledge, this is the first report to investigate potential changes in $\mathrm{CDX} 2$ expression during CRC development and chemotherapy.

Previous reports utilized TMAs to analyze CDX2 expression in CRCs [23, 27-30]. One major concern of TMA analysis is that the small core samples may not be representative of the entire tumor $[31,32]$. Our results demonstrating the heterogeneity of $\mathrm{CDX} 2$ expression in primary CRCs raise the possibility that previous studies reporting CDX2 expression using TMAs might not reflect the status of whole CRC sections. Conversely, an optimal cut-off value to determine the CDX2 expression status in whole CRC sections might be necessary if $\mathrm{CDX} 2$ expression determined by TMAs is utilized in daily medical practice as a prognostic or predictive biomarker for response to chemotherapy.

We hypothesized that the percentage of the CDX2negative component in the primary CRC would increase during cancer evolution, based on recent studies showing that the loss of CDX2 expression was associated with worse prognosis [16, 18, 19]. However, our results demonstrated that the differences in CDX2 expression status between primary CRCs and their corresponding liver metastases were minimal. Additionally, the numbers of liver metastases were comparable between the CDX2positive and -negative groups. These results suggested that while CDX2 might not play an important role in the development of liver metastasis of CRC, and the CDX2 expression status might be useful as a biomarker for the clinical evaluation of both primary CRC lesions and liver metastases.

The utility of CDX2 expression status as a predictive biomarker for response to chemotherapy remains controversial. In contrast to the suggested benefit of adjuvant chemotherapy for stage II and stage III CDX2-negative CRCs [16], the benefit of systemic chemotherapy was significantly lower for CDX2-negative metastatic CRCs than for CDX2-positive CRCs [19]. Generally, in tumors with heterogeneous components 
Table 3: Concordance of CDX2 expression between primary colorectal cancers and corresponding liver metastases in patients with and without chemotherapy

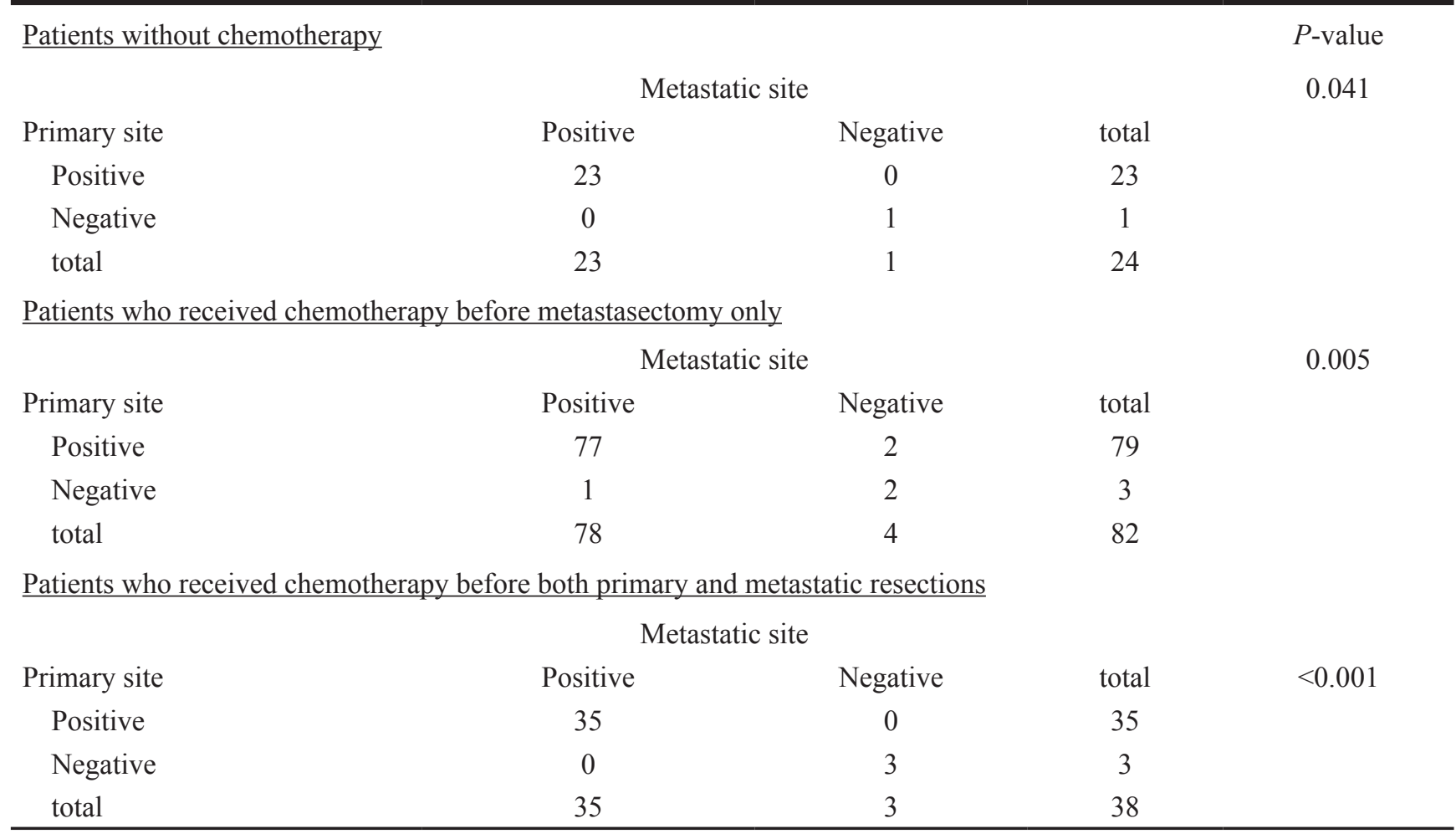

exhibiting distinct sensitivities to chemotherapy, the ratio of components conferring chemotherapy resistance increased during treatment [33]. In the present study, we found that the CDX2 expression status in liver metastases changed minimally from that in primary lesions, indicating that the CDX2 expression status might not be directly associated with chemotherapy sensitivity. Our retrospective study assessing the association between CDX2 expression status and chemotherapy sensitivity warrants further validation.

Our study has several limitations due mainly to its retrospective nature. First, we selected the patients who underwent primary CRC resection and liver metastasectomy in the current study. However, we believe that selection bias was negligible because the comparisons included primary CRC and metastatic samples that were obtained from the same patients, and the prevalence CDX2 expression loss of $4.9 \%$ was similar to those reported previously $[16,19]$. Second, long-term storage of samples (3-10 years) might have affected CDX2 immunoreactivity. However, the year of diagnosis did not have a significant impact on the CDX2 expression (data not shown), suggesting that the storage period did not substantially affect CDX2 immunoreactivity. Third, the number of CDX2-negative CRC patients was relatively small, and information about CDX2 expression alterations in the CDX2-negative CRCs was limited. This is because
CDX2-negative CRCs are rare, and only Japanese patients at a single cancer hospital were included in the present study. However, in this study, on an examination of cross-sections, primary CRC revealed heterogenous CDX2 expression, and 21.9\% (30/137) of CDX2-positive primary CRCs also had CDX2-negative cancer cells in the tumor (Figure 2). Thus, the high concordance rate of the CDX2 expression status was achieved through expression consistency in both CDX2-positive and CDX2-negative components of the primary tumor. Although our primary hypothesis regarding the association between CRCs and CDX2 expression status was whether the alterations from CDX2-positive to CDX2-negative during tumor progression or chemotherapy were negligible, the investigation of the opposite situation also yields valuable information. In survival analysis, the number of patients with CDX2-negative CRCs is small, and the results might not be sufficiently powered to detect a difference. Further studies with a large sample size are necessary to elucidate the impact of chemotherapy or tumor progression in CDX2-negative CRCs and confirm our findings.

This is the first study to demonstrate that the CDX2 expression status in CRCs was highly concordant between primary CRCs and their corresponding liver metastases, independent of chemotherapy, suggesting that the CDX2 expression status in CRC was not affected by metastasis or chemotherapy. 


\section{MATERIALS AND METHODS}

\section{Patients and tissue samples}

In this retrospective study, CRC surgical samples were obtained from 144 consecutive patients who underwent colorectal resection with extended lymph node dissection (D2 or D3) and liver resection for CRC at the Cancer Institute Hospital in Tokyo, Japan, between 2006 and 2014. Only patients with resected tumor samples available from both primary CRCs and corresponding liver metastases that were suitable for immunohistochemical analysis were included. In total, 288 samples consisting of primary CRCs and corresponding liver metastases from 144 patients were included. Clinical data and histological features included age, sex, tumor location, number of liver metastases, clinical-stage, history of chemotherapy, and tumor grade. Histological findings were determined according to the fourth edition of the World Health Organization criteria [34]. Tumor stage was determined according to the seventh edition of the tumor-node-metastasis staging manual of the American Joint Committee on Cancer [35]. Patients who received chemotherapy within six months before surgical resection were defined as those with a history of chemotherapy. Patients who did not give written informed consent were excluded from this study. Whole CRC sections were reviewed and confirmed by two of the investigators (Y.S. and K.I.). The study was approved by the committee for ethics at the Cancer Institute Hospital (No. 2016-1087).

\section{Immunohistochemical analysis}

IHC was performed using 4- $\mu \mathrm{m}$ thick formalin-fixed paraffin-embedded CRC sections. Sections of the largest cross-sectional slice of the primary and metastatic lesions were used for the analysis. We used a primary mouse antihuman CDX2 monoclonal antibody (clone DAK-CDX2, 1:100 dilution, DAKO, Carpinteria, CA, USA) that was previously validated for diagnostic applications [9]. All samples were handled in an anonymous fashion. Tissue slides were stained using a Bond-III automatic stainer (Leica Microsystems, Buffalo Grove, IL, USA), and antigen detection was achieved using the Bond Polymer Refine detection kit (Leica Microsystems).

CRC diagnosis was confirmed by two experienced pathologists (Y.S. and K.I.) based on the microscopic examination of hematoxylin-eosin-stained slides. All cases were scored blindly by two experienced pathologists (Y.S. and K.I.) in an independent fashion. Cases that were difficult to interpret immunohistochemically were discussed with a third experienced pathologist (H.K.). Nuclear CDX2 expression was scored based on the number of CDX2-positive tumor cells.

In the secondary analysis, 268 matched samples from 134 patients used the following primary antibodies: anti- human CK7 monoclonal antibody (clone OV-TL 12/30, 1:200 dilution, DAKO, Carpinteria, CA, USA), anti-human CK20 monoclonal antibody (clone IT-Ks 20.8, 1:100 dilution, Progen Biotechnik, Heidelberg, Germany), anti-human CEA monoclonal antibody (clone COL-1, ready-to-use prediluted, Nichirei Bioscience, Tokyo, Japan), and anti-human MUC2 monoclonal antibody (clone Ccp58, 1:200 dilution, Leica Biosystems, Newcastle, UK). As for CDX1 IHC, anti-human CDX1 polyclonal antibody (HPA055196, 1:500 dilution, Atlas Antibodies AB, Stockholm, Sweden) and anti-rabbit IgG polyclonal antibody (211-005-109, Jackson ImmunoResearch Laboratories, Inc., West Grove, PA, USA) were used.

\section{Statistical analysis}

All statistical analyses were conducted using $\mathrm{R}$ version 3.2.2 (R Foundation for Statistical Computing, Vienna, Austria). Fisher's exact test was used to analyze the association between primary CRCs and corresponding liver metastases based on the CDX2 expression status and to evaluate differences in relative frequencies of clinicopathological parameters between the CDX2positive and -negative groups. Cohen's kappa index was used to assess the concordance of CDX2 expression status determined by the two investigators [36]. Differences between mean values of two groups were evaluated by the Mann-Whitney $U$ test. Two-tailed $P$ values of 0.05 or less were considered to be statistically significant. For the survival analysis between the patient subgroups, we used Kaplan-Meier curve and log-rank test. RFS and OS were calculated from the date of liver metastasectomy to the date of the first recurrence at any site or death.

\section{Abbreviations}

CRC: colorectal cancer; CDX2: caudal-type homeobox transcription factor 2; IHC: immunohistochemistry; TMA: tissue microarray; CK: cytokeratin; MUC2: mucin 2; CEA: carcinoembryonic antigen; CDX1: caudal-type homeobox transcription factor 1 .

\section{Author contributions}

Y.S. contributed to the conception of the study and drafted the manuscript. Y.S., K.I., and H.K. reviewed pathological diagnosis of all specimens and performed immunohistochemical analysis. E.R. performed statistical analysis. Y.M. and A.S. collected clinical information and obtained informed consent from patients. N.Y. and Y.I. provided whole primary colorectal cancer sections. All authors gave final approval for publication.

\section{ACKNOWLEDGMENTS}

We thank Mr. Motoyoshi Iwakoshi and Ms. Tomoyo Kakita for their kind assistance in sample preparation 
and Drs. Yutaka Takazawa, Hiroshi Kawachi, Manabu Takamatsu, and Maki Kobayashi for their constructive comments on the study.

\section{CONFLICTS OF INTEREST}

The authors declare that no competing interests exist.

\section{FUNDING}

This work was supported by Japan Society for the Promotion of Science KAKENHI Grant Number JP16K21590 and Grants-in-Aid from the Foundation for the Promotion of Cancer Research in Japan. None of the funding sources had a role in the study design, data collection/analysis, interpretation of data, or writing of the manuscript.

\section{REFERENCES}

1. Gao N, White P, Kaestner KH. Establishment of intestinal identity and epithelial-mesenchymal signaling by $\mathrm{Cdx} 2$. Dev Cell. 2009; 16:588-99. https://doi.org/10.1016/j. devcel.2009.02.010.

2. Silberg DG, Swain GP, Suh ER, Traber PG. Cdx1 and cdx2 expression during intestinal development. Gastroenterology. 2000; 119:961-71.

3. Hryniuk A, Grainger S, Savory JG, Lohnes D. Cdx function is required for maintenance of intestinal identity in the adult. Dev Biol. 2012; 363:426-37. https://doi.org/10.1016/j. ydbio.2012.01.010.

4. Grainger S, Savory JG, Lohnes D. Cdx2 regulates patterning of the intestinal epithelium. Dev Biol. 2010; 339:155-65. https://doi.org/10.1016/j.ydbio.2009.12.025.

5. Suh E, Chen L, Taylor J, Traber PG. A homeodomain protein related to caudal regulates intestine-specific gene transcription. Mol Cell Biol. 1994; 14:7340-51.

6. Wicking C, Simms LA, Evans T, Walsh M, Chawengsaksophak K, Beck F, Chenevix-Trench G, Young J, Jass J, Leggett B, Wainwright B. CDX2, a human homologue of Drosophila caudal, is mutated in both alleles in a replication error positive colorectal cancer. Oncogene. 1998; 17:657-9. https://doi.org/10.1038/sj.onc.1201971.

7. Saad RS, Ghorab Z, Khalifa MA, Xu M. CDX2 as a marker for intestinal differentiation: Its utility and limitations. World J Gastrointest Surg. 2011; 3:159-66. https://doi. org/10.4240/wjgs.v3.i11.159.

8. Werling RW, Yaziji H, Bacchi CE, Gown AM. CDX2, a highly sensitive and specific marker of adenocarcinomas of intestinal origin: an immunohistochemical survey of 476 primary and metastatic carcinomas. Am J Surg Pathol. 2003; 27:303-10.
9. Borrisholt M, Nielsen S, Vyberg M. Demonstration of CDX2 is highly antibody dependant. Appl Immunohistochem Mol Morphol. 2013; 21:64-72. https://doi.org/10.1097/ PAI.0b013e318257f8aa.

10. Barbareschi M, Murer B, Colby TV, Chilosi M, Macri E, Loda M, Doglioni C. CDX-2 homeobox gene expression is a reliable marker of colorectal adenocarcinoma metastases to the lungs. Am J Surg Pathol. 2003; 27:141-9.

11. Abate-Shen C. Deregulated homeobox gene expression in cancer: cause or consequence? Nat Rev Cancer. 2002; 2:777-85. https://doi.org/10.1038/nrc907.

12. Gerlinger M, Rowan AJ, Horswell S, Math M, Larkin J, Endesfelder D, Gronroos E, Martinez P, Matthews N, Stewart A, Tarpey P, Varela I, Phillimore B, et al. Intratumor heterogeneity and branched evolution revealed by multiregion sequencing. N Engl J Med. 2012; 366:883-92. https://doi.org/10.1056/NEJMoa1113205.

13. Choi CR, Bakir IA, Hart AL, Graham TA. Clonal evolution of colorectal cancer in IBD. Nat Rev Gastroenterol Hepatol. 2017; 14:218-29. https://doi.org/10.1038/nrgastro.2017.1.

14. Nowell PC. The clonal evolution of tumor cell populations. Science. 1976; 194:23-8.

15. Greaves M, Maley CC. Clonal evolution in cancer. Nature. 2012; 481:306-13. https://doi.org/10.1038/nature10762.

16. Dalerba P, Sahoo D, Paik S, Guo X, Yothers G, Song N, Wilcox-Fogel N, Forgo E, Rajendran PS, Miranda SP, Hisamori S, Hutchison J, Kalisky T, et al. CDX2 as a Prognostic Biomarker in Stage II and Stage III Colon Cancer. N Engl J Med. 2016; 374:211-22. https://doi. org/10.1056/NEJMoa1506597.

17. Hinoi T, Tani M, Lucas PC, Caca K, Dunn RL, Macri E, Loda M, Appelman HD, Cho KR, Fearon ER. Loss of CDX2 expression and microsatellite instability are prominent features of large cell minimally differentiated carcinomas of the colon. Am J Pathol. 2001; 159:2239-48. https://doi.org/10.1016/S0002-9440(10)63074-X.

18. Baba Y, Nosho K, Shima K, Freed E, Irahara N, Philips J, Meyerhardt JA, Hornick JL, Shivdasani RA, Fuchs CS, Ogino S. Relationship of CDX2 loss with molecular features and prognosis in colorectal cancer. Clin Cancer Res. 2009; 15:4665-73. https://doi.org/10.1158/1078-0432. CCR-09-0401.

19. Zhang BY, Jones JC, Briggler AM, Hubbard JM, Kipp BR, Sargent DJ, Dixon JG, Grothey A. Lack of CaudalType Homeobox Transcription Factor 2 Expression as a Prognostic Biomarker in Metastatic Colorectal Cancer. Clin Colorectal Cancer. 2017; 16:124-8. https://doi.org/10.1016/j. clcc.2016.09.003.

20. Adam R, Wicherts DA, de Haas RJ, Ciacio O, Levi F, Paule B, Ducreux M, Azoulay D, Bismuth H, Castaing D. Patients with initially unresectable colorectal liver metastases: is there a possibility of cure? J Clin Oncol. 2009; 27:1829-35. https://doi.org/10.1200/JCO.2008.19.9273. 
21. NIH consensus conference. Adjuvant therapy for patients with colon and rectal cancer. JAMA. 1990; 264:1444-50.

22. Ayadi M, Bouygues A, Ouaret D, Ferrand N, Chouaib S, Thiery JP, Muchardt C, Sabbah M, Larsen AK. Chronic chemotherapeutic stress promotes evolution of stemness and WNT/beta-catenin signaling in colorectal cancer cells: implications for clinical use of WNT-signaling inhibitors. Oncotarget. 2015; 6:18518-33. https://doi.org/10.18632/ oncotarget.3934.

23. Bae JM, Lee TH, Cho NY, Kim TY, Kang GH. Loss of CDX2 expression is associated with poor prognosis in colorectal cancer patients. World J Gastroenterol. 2015; 21:1457-67. https://doi.org/10.3748/wjg.v21.i5.1457.

24. Freund JN, Domon-Dell C, Kedinger M, Duluc I. The Cdx-1 and Cdx-2 homeobox genes in the intestine. Biochem Cell Biol. 1998; 76:957-69.

25. Lynch J, Keller M, Guo RJ, Yang D, Traber P. Cdx1 inhibits the proliferation of human colon cancer cells by reducing cyclin D1 gene expression. Oncogene. 2003; 22:6395-407. https://doi.org/10.1038/sj.onc.1206770.

26. Guo RJ, Huang E, Ezaki T, Patel N, Sinclair K, Wu J, Klein P, Suh ER, Lynch JP. Cdx1 inhibits human colon cancer cell proliferation by reducing beta-catenin/T-cell factor transcriptional activity. J Biol Chem. 2004; 279:36865-75. https://doi.org/10.1074/jbc.M405213200.

27. Minoo P, Zlobec I, Peterson M, Terracciano L, Lugli A. Characterization of rectal, proximal and distal colon cancers based on clinicopathological, molecular and protein profiles. Int J Oncol. 2010; 37:707-18.

28. Winn B, Tavares R, Fanion J, Noble L, Gao J, Sabo E, Resnick MB. Differentiating the undifferentiated: immunohistochemical profile of medullary carcinoma of the colon with an emphasis on intestinal differentiation. Hum Pathol. 2009; 40:398-404. https://doi.org/10.1016/j. humpath.2008.08.014.

29. Dawson H, Galvan JA, Helbling M, Muller DE, Karamitopoulou E, Koelzer VH, Economou M, Hammer
C, Lugli A, Zlobec I. Possible role of Cdx2 in the serrated pathway of colorectal cancer characterized by BRAF mutation, high-level $\mathrm{CpG}$ Island methylator phenotype and mismatch repair-deficiency. Int J Cancer. 2014; 134 : 2342-51. https://doi.org/10.1002/ijc.28564.

30. Karamitopoulou E, Zlobec I, Panayiotides I, Patsouris ES, Peros G, Rallis G, Lapas C, Karakitsos P, Terracciano LM, Lugli A. Systematic analysis of proteins from different signaling pathways in the tumor center and the invasive front of colorectal cancer. Hum Pathol. 2011; 42:1888-96. https://doi.org/10.1016/j.humpath.2010.06.020.

31. Hsu FD, Nielsen TO, Alkushi A, Dupuis B, Huntsman D, Liu CL, van de Rijn M, Gilks CB. Tissue microarrays are an effective quality assurance tool for diagnostic immunohistochemistry. Mod Pathol. 2002; 15:1374-80. https://doi.org/10.1097/01.MP.0000039571.02827.CE.

32. Kononen J, Bubendorf L, Kallioniemi A, Barlund M, Schraml P, Leighton S, Torhorst J, Mihatsch MJ, Sauter G, Kallioniemi OP. Tissue microarrays for high-throughput molecular profiling of tumor specimens. Nat Med. 1998; $4: 844-7$.

33. Holohan C, Van Schaeybroeck S, Longley DB, Johnston PG. Cancer drug resistance: an evolving paradigm. Nat Rev Cancer. 2013; 13:714-26. https://doi.org/10.1038/nrc3599.

34. Bosman FT, Carneiro F, Hruban RH, Theise ND. WHO Classification of Tumours of the Digestive System 4th ed. World Health Organization. 2010.

35. Edge SB, Compton CC. The American Joint Committee on Cancer: the 7th Edition of the AJCC Cancer Staging Manual and the Future of TNM. Ann Surg Oncol. 2010; 17:1471-4. https://doi.org/10.1245/s10434-010-0985-4.

36. Sim J, Wright CC. The kappa statistic in reliability studies: use, interpretation, and sample size requirements. Phys Ther. 2005; 85:257-68. 